\title{
Approximating Minimum-Weight Triangulations in Three Dimensions*
}

\author{
B. Aronov ${ }^{1}$ and S. Fortune ${ }^{2}$ \\ ${ }^{1}$ Computer and Information Science, Polytechnic University, \\ Brooklyn, NY 11201, USA \\ aronov@ziggy.poly.edu \\ 2 Bell Laboratories, \\ Murray Hill, NJ 07974, USA \\ sjf@research.bell-labs.com
}

\begin{abstract}
Let $S$ be a set of noncrossing triangular obstacles in $\mathbb{R}^{3}$ with convex hull $H$. A triangulation $\mathcal{T}$ of $H$ is compatible with $S$ if every triangle of $S$ is the union of a subset of the faces of $\mathcal{T}$. The weight of $\mathcal{T}$ is the sum of the areas of the triangles of $\mathcal{T}$. We give a polynomial-time algorithm that computes a triangulation compatible with $S$ whose weight is at most a constant times the weight of any compatible triangulation.

One motivation for studying minimum-weight triangulations is a connection with ray shooting. A particularly simple way to answer a ray-shooting query ("Report the first obstacle hit by a query ray") is to walk through a triangulation along the ray, stopping at the first obstacle. Under a reasonably natural distribution of query rays, the average cost of a ray-shooting query is proportional to triangulation weight. A similar connection exists for line-stabbing queries ("Report all obstacles hit by a query line").
\end{abstract}

\section{Introduction}

Let $S$ be a finite set of noncrossing obstacles (line segments in $\mathbb{R}^{2}$, triangles in $\mathbb{R}^{3}$ ) with convex hull $H$. A triangulation $\mathcal{T}$ of $H$ is compatible with $S$ if each obstacle is the union of a subset of the faces of $\mathcal{T}$. $\mathcal{T}$ may have Steiner vertices, i.e., vertices that are not vertices of $S$. The weight of a facet $f,|f|$, is edge length in $\mathbb{R}^{2}$ and triangle area in $\mathbb{R}^{3}$ ); the weight of $\mathcal{T},|\mathcal{T}|$, is the sum of the weights of its facets.

We give a polynomial-time algorithm that computes a triangulation $\mathcal{T}$ compatible with a three-dimensional obstacle set $S$. The weight of $\mathcal{T}$ is within a constant factor

\footnotetext{
* Work of the first author was performed while he was visiting Bell Laboratories, Murray Hill, NJ, USA.
} 
of the smallest possible compatible triangulation. The algorithm is a generalization of Eppstein's algorithm [9] to compute a constant-factor approximation to the minimumlength Steiner triangulation of a set of points in two dimensions. As with Eppstein's analysis, the approximation ratio is large, though constant.

The algorithm has two steps. The first step produces a depth-bounded octtree from the obstacles, where the rule is that an octtree cube is split if it meets an obstacle vertex or edge and is not too small. The second step triangulates each leaf cube in a manner compatible with the obstacles and neighboring leaf cubes. For the worst-case set of $n$ obstacles, the algorithm runs in time $O\left(n^{6}\right)$ and produces as many tetrahedra. An improvement of an order of magnitude in both running time and number of tetrahedra is possible if the obstacle set is well-shaped (see Section 3.3). A further improvement is possible if obstacles are just points (though this is not discussed in this paper).

The proof of the approximation ratio has two parts. The first part is to show that the total surface area of the octtree is at most a constant factor times the area of an arbitrary triangulation $\hat{\mathcal{T}}$. To do this we charge the surface area of each leaf cube to some local feature of $\hat{\mathcal{T}}$. There are essentially two cases: if the central subcube of the leaf cube meets a vertex, edge, or face of $\hat{\mathcal{T}}$, then the area of $\hat{\mathcal{T}}$ within the leaf cube must be at least proportional to its surface area. Otherwise the central subcube must be contained in a tetrahedron of $\hat{\mathcal{T}}$, some face of which must have area at least proportional to the surface area of the leaf cube. (The actual argument is more complex, to guarantee that a single tetrahedral face is not charged by too many leaf cubes.) The second part of the approximation-ratio bound is to triangulate all leaf cubes with total area at most a constant times the tree surface area plus the obstacle area.

We also briefly consider the two-dimensional case of compatible triangulations. As in three dimensions, there is a polynomial-time algorithm that approximates the minimumweight compatible triangulation. In Section 2 we show that the minimal Steiner triangulation weight is approximately the length of $S$ plus the length of the MST (within a logarithmic factor). Here MST is the minimum Steiner spanning tree of $S$, i.e., the minimum-length set of line segments so that MST $\cup S$ is connected.

Other Work. A long-standing open problem is the question of whether there is a polynomial-time algorithm that finds the minimum-length triangulation of a point set in two dimensions, without using additional Steiner points. Beirouti and Snoeyink [5] report recent progress and give many references. Eppstein [9] describes a polymial-time algorithm that gives a constant-factor approximation to the minimum-weight triangulation of a two-dimensional point set, allowing Steiner points.

Average-Case Line Stabbing and Ray Shooting. We now describe a connection between triangulation weight and the average cost of simple algorithms for ray shooting ("Report the first obstacle hit by a ray") and line stabbing ("Report all obstacle hit by a line").

Line-stabbing queries can be answered in a particularly simple way given a triangulation of space compatible with the obstacles. It suffices to walk through the triangulation along the line, reporting each encountered obstacle. The walk takes constant time per visited triangle, so the total cost of the walk is proportional to the number of triangle faces crossed. In the worst case, the walk can be long. For example, Agarwal et al. [2] describe a configuration of $n$ obstacle triangles in $\mathbb{R}^{3}$ so that there is a line missing all 
the obstacles yet hitting $\Omega(n)$ faces of any triangulation compatible with the obstacles.

We consider instead the average-case cost of line stabbing, using the standard rigidmotion invariant measure $\mu$ on lines [16]. Let $L(U)$ be the set of lines that meet a set $U$. A basic fact from integral geometry is that for a facet $f, \mu(L(f))$ is the weight of $f$ (length in two dimensions, area in three dimensions) times a constant depending upon dimension.

Let $S$ be a set of obstacles so that any facet of its convex hull is the union of obstacles. Let $\mathcal{T}$ be a Steiner triangulation compatible with $S$. For line $\ell$, let $s(\ell)$ and $t(\ell)$ be the number of obstacle facets and triangulation facets intersected by $\ell$, respectively. Then

$$
\frac{\int t(\ell) d \mu}{\mu(L(H))}
$$

is the average walk length to answer a line-stabbing query that meets the convex hull $H$ of $S$. The ratio

$$
c(\mathcal{T})=\frac{\int t(\ell) d \mu}{\int s(\ell) d \mu}
$$

is the average walk length per reported obstacle facet. Letting $\delta_{f}(l)$ be 1 if line $l$ hits facet $f$ and 0 otherwise, we have that

$$
c(\mathcal{T})=\frac{\int t(\ell) d \mu}{\int s(\ell) d \mu}=\frac{\sum_{f \in \mathcal{T}} \int \delta_{f}(l) d \mu}{\sum_{f \in \mathcal{S}} \int \delta_{f}(l) d \mu}=\frac{\sum_{f \in \mathcal{T}}|f|}{\sum_{f \in S}|f|}=\frac{|\mathcal{T}|}{|S|} .
$$

A ray-shooting query can be answered by an algorithm similar to the line-stabbing algorithm: locate the endpoint of the ray in the triangulation, and walk along the ray through the triangulation until an obstacle facet is encountered. For a ray $r$, let $t(r)$ be the number of triangulation facets encountered in a walk along $r$ up to an obstacle. Formally, $t(r)$ should include the facet containing the ray endpoint (if any) and should count all facets up to but not including the first obstacle facet. Clearly, $t(r)$ is proportional to the cost of answering a ray-shooting query, ignoring the cost of locating the triangle containing the endpoint of $t$.

The ratio $c(\mathcal{T})$ is also the average value of $t(r)$, for a particular distribution of query rays. Let $\ell$ be a directed line, and assume that the intersection of $\ell$ with each obstacle is either empty or a point. (Note that $c(\mathcal{T})$ is changed neither by assuming that lines are directed, nor by ignoring the measure-zero set of lines that overlap an obstacle.) Associate with $\ell$ the set of rays in the same direction as $\ell$ and with endpoints at an intersection of $\ell$ with an obstacle. Clearly, $s(\ell)$ is the number of such rays and $t(\ell)=\sum t(r), r$ in the associated set of rays. Hence

$$
c(\mathcal{T})=\frac{\int t(\ell) d \mu}{\int s(\ell) d \mu}
$$

is the average of $t(r)$, under the distribution on rays induced by the distribution on lines. Using integral geometry [16, Section 12.7, eq. (12.60)], the induced ray distribution is $\sin \theta d A \wedge d u$, where $d A$ is the uniform area distribution, $d u$ is the uniform solid angle distribution, and $\theta$ is the angle between the ray and the surface of the obstacle. Informally, a ray is chosen with endpoint uniformly at random from an obstacle and with direction proportional to the sine of the angle $\theta$ between the ray and the obstacle. 
Let

$$
c(S)=\inf _{\hat{\mathcal{T}}} c(\hat{\mathcal{T}})
$$

where $\hat{\mathcal{T}}$ ranges over all triangulations compatible with $S$. Clearly, to answer line-stabbing or ray-shooting queries it is desirable to choose a triangulation $\mathcal{T}$ with $c(\mathcal{T})$ as close as possible to $c(S)$. It is not obvious that the lower bound $c(S)$ can be attained [6]; for example, it is conceivable that it is always possible to decrease weight and hence $c(\mathcal{T})$ by adding Steiner points.

In two dimensions, $c(S) \approx(|\mathrm{MST}|+|S|) /|S|$ (see Section 2). Hence in cases where the MST is short, for example if the obstacle set is connected or nearly connected, the average cost of ray-shooting by walking through a triangulation should be small. This good behavior has been observed experimentally [10] (even without explicitly minimizing the weight of the triangulation).

Other Work. The ray-shooting problem has been studied extensively in computational geometry (see [1] or [15] for a survey of theoretical results). Assuming roughly linear data-structure storage, the best theoretical algorithms for ray shooting have worst-case query time $O(\log n)$ for a simple polygon [11], roughly $O(\sqrt{n})$ for a set of planar linesegments [4], and roughly $O\left(n^{3 / 4}\right)$ for a set of triangles in three dimensions [3]. The last two query times can be improved to $O(\log n)$ with polynomial storage. Agarwal et al. [2] consider the line-stabbing number of triangulations consistent with a set of obstacles. Mitchell et al. [12] consider segment shooting, a variant of ray shooting. They show that the cost of a segment-shooting query in an octtree can be bounded up to a constant factor by the "cover complexity" of the segment.

\section{The Two-Dimensional Case}

Throughout this section $S$ is a set of $n$ planar obstacle segments that meet only at endpoints; $S$ must include segments partitioning the boundary of its convex hull. A triangulation $\mathcal{T}$ is compatible with $S$ if any edge in $S$ is the union of closed edges of $\mathcal{T}$. Vertical bars $|\cdot|$ denote length, thus $|S|$ is the sum of the lengths of the segments in $S$. Let $M$ be $\inf _{\mathcal{T}}|\mathcal{T}|$, where $\mathcal{T}$ varies over triangulations compatible with $S$.

Lemma 2.1. In polynomial time it is possible to compute a polynomial-size Steiner triangulation $\mathcal{T}$ compatible with $S$ so that $|\mathcal{T}|=O(M)$.

We omit a detailed proof of Lemma 2.1; it can be proven using techniques of Eppstein [9] or of the proof in Section 3. The basic strategy is to build a depth-bounded balanced quadtree using only the vertices of $S$, and then triangulate each square in a fashion compatible with the edges of $S$ meeting the square. We remark that Lemma 2.1 depends upon including the length of the obstacle set $S$ in the length of the triangulation; 
if the length is not included, a constant-factor polynomial-time approximation algorithm is not known [9].

Let MST be a minimum (Steiner) spanning tree of $S$, i.e., a set of segments of minimum total length so that $S \cup \mathrm{MST}$ is connected. An easy compactness argument shows that the minimum length can actually be attained.

Lemma 2.2. $|S|+|\mathrm{MST}| \leq M \leq O((|S|+|\mathrm{MST}|) \log n)$.

Proof. The first inequality is obvious, since any triangulation compatible with $S$ must be connected. For the second, notice that MST $\cup S$ partitions the convex hull of $S$ into simple polygons (recall that $S$ includes the convex hull boundary). The total number of vertices is $O(n)$, since the MST is a forest with no vertices of degree 2 and the number of leaves is the number of connected components of $S$. By a result of Clarkson [7], each simple polygon can be triangulated with weight proportional to $\log n$ times the perimeter of the polygon.

Let $D$ be the diameter of $S$, i.e., the length of the longest segment contained in the convex hull of $S$. The minimum spanning tree of the vertices of $S$ has length at most $O(D \sqrt{n})$, hence $|\mathrm{MST}| \leq O(D \sqrt{n})$. The following lemma improves the worst-case bound that can be obtained from Lemma 2.2 and this estimate by a factor of $\log n$. Again we omit a detailed proof of the lemma (see Section 3.7 for a similar proof in three dimensions).

Lemma 2.3. $M \leq O(|S|+D \sqrt{n})$.

Corollary 2.4. $|\mathrm{MST}| /|S|+1 \leq c(S) \leq \min (O((1+|\mathrm{MST}| /|S|) \log n), O(1+$ $D \sqrt{n} /|S|))$.

\section{The Three-Dimensional Case}

This section describes an algorithm that produces a Steiner triangulation compatible with a set of polyhedral obstacles in three dimensions. The triangulation has area within a constant factor of the smallest possible. Section 3.1 reviews some basic definitions. Section 3.2 states the main theorem in the case where the obstacles are "wide," a condition on the aspect ratio of their convex hull. The main theorem is proved in Sections 3.33.5. Section 3.3 gives an algorithm that constructs an octtree from a set of polyhedral obstacles. In Section 3.4 we prove that the surface area of the octtree is at most a constant factor times the area of any Steiner triangulation compatible with the obstacles, while Section 3.5 gives an algorithm to triangulate the octtree with total area proportional to the area of the octtree plus the area of the obstacles. The wideness condition on obstacles is removed in Section 3.6. Finally in Section 3.7 we show some worst-case bounds on the ratio of triangulation area to obstacle area. 


\subsection{Definitions}

We use terminology from the theory of convex polyhedra [8]. For example, a polyhedral set is any set obtained from open and closed halfspaces by a finite number of unions and intersections. A face of a convex polyhedron $C$ is the relative interior of the intersection of the closure of $C$ with a hyperplane supporting $C ; \partial C$ is the relative boundary of $C$.

A polyhedral subdivision $\mathcal{Q}$ of a polyhedral set $Q$ is a finite partition of $Q$ into relatively open convex polyhedral cells so that every face of every cell is a union of cells in $\mathcal{Q}$. A subdivision is proper if every face of every cell is itself a cell in the subdivision. Not all subdivisions are proper, since some face of a cell may be subdivided into more than one cell. If $f$ is a face of a cell $C$, we will occasionally refer to $f$ as a polyhedral face of $C$ to distinguish it from a cell contained in $\partial C$ that is not a face of $C$. The $k$-skeleton $\mathcal{Q}^{(k)}$ of $\mathcal{Q}$ is the subdivision consisting of all cells of $\mathcal{Q}$ of dimension at most $k$. If $C$ is a convex polytope that is the union of cells of $\mathcal{Q}$, then $\operatorname{bdry}(C, \mathcal{Q})$ is the subdivision consisting of the cells in $\mathcal{Q}$ whose union is $\partial C$. For convenience we define $\operatorname{bdry}(\mathcal{Q})$ to be $\operatorname{bdry}(Q, \mathcal{Q})$. The area of a subdivision $\mathcal{Q}$, $\operatorname{area}(\mathcal{Q})$, is the sum of the areas of the 2-cells in $\mathcal{Q}$; similarly the length of $\mathcal{Q}$, length $(\mathcal{Q})$, is the sum of the lengths of the 1-cells in $\mathcal{Q}$. A triangulation of a polyhedral set is a proper polyhedral subdivision so that all cells are simplices. A triangulation $\mathcal{T}$ is compatible with a subdivision $\mathcal{S}$ if every cell in $\mathcal{S}$ is a union of cells in $\mathcal{T}$.

If $C$ is a square or cube and $k$ a positive real, then $k C$ is the square or cube with the same center and orientation as $C$ and side length $k$ times the side length of $C$. We write for example $C / 2$ for $(1 / 2) C$.

\subsection{The Main Theorem for Wide Obstacles}

The obstacle set $S$ is a subdivision in $\mathbb{R}^{3}$ consisting only of 0 -, 1-, and 2 -simplices. For simplicity we assume that there are no isolated edges and vertices in $S$, i.e., every edge or vertex lies on the boundary of a 2-simplex; with minor modifications the algorithm can be extended to handle isolated edges and vertices. We let $H$ be the convex hull of $S$; we assume that $S$ contains a triangulation of $\partial H$. Throughout $n$ is the total number of simplices in $S$.

Let $B$ be the smallest cube containing $S$, and let its side length be $b$. Obstacle set $S$ is wide if the area of $\partial H$ is $\Omega\left(b^{2}\right)$. Informally, $H$ can look like a ball or pancake, but not like a pencil. The following theorem holds for wide obstacles; it follows immediately from Theorem 3.2 in Section 3.4 and Theorem 3.9 in Section 3.5, using the fact that the area of $\partial H$ and hence $S$ is $\Omega\left(b^{2}\right)$. The wideness assumption is removed in Section 3.6.

Theorem 3.1. Let $S$ be a wide set of obstacles with a total of $n$ simplices. In time $O\left(n^{5}\right)$ it is possible to compute a triangulation compatible with $S$ whose area is within a constant factor of the smallest possible. The triangulation has $O\left(n^{5}\right)$ tetrahedra and partitions the convex hull of $S$.

\subsection{The Octtree Algorithm}

We now describe how to build an octtree $T$ from the obstacle set $S$. If necessary, perturb the minimal enclosing cube $B$ slightly so that no obstacle face is parallel to a face of $B$. 
Recall that $b$ is the side length of $B$. Let $m$ be the first power of two greater than or equal to $n$. Define $s_{0}=b / m$. Then $b / s_{0}$ is a power of two and $b /(2 n)<s_{0} \leq b / n$.

We first build an octtree $T_{0}$. Every node of the tree is a cube. $B$ is the root node. A cube is skewered if it meets the 1 -skeleton of $S$. A cube $C$ is subdivided (i.e., is not a leaf) if it is skewered and has side length greater than $s_{0}$; its children are the eight subcubes obtained by cutting it by the three planes through its center parallel to its facets. This process is repeated until no cube can be further subdivided. Clearly, each leaf cube in the octtree has side length $2^{i} s_{0}$, for some integral $i \geq 0$, and each skewered leaf cube has side length $s_{0}$.

Octtree $T_{0}$ must then be made balanced, resulting in the octtree $T$. Two cubes are adjacent if they have overlapping 2-faces; an octtree is balanced if the side lengths of any two adjacent leaf cubes differ by at most two. If an octtree is unbalanced, it can be made balanced by repeatedly choosing a pair of adjacent cubes that violate the balance property and subdividing the larger cube. Standard results [13] imply that balancing $T$ increases the number of cubes by at most a constant factor.

An obstacle triangle $\Delta$ fully cuts a cube $C$ if $\Delta$ intersects $C$ and $\partial \Delta$ avoids $C$. Notice that a cube may be fully cut by an obstacle triangle independently of whether or not it is skewered.

\subsection{Area of the Octtree}

The area of octtree $T$, area $(T)$, is $\sum_{C}$ area $(\partial C \cap H)$, where $C$ varies over the leaf cubes of $T$. Let $M=\inf _{\hat{\mathcal{T}}}$ area $(\hat{\mathcal{T}})$, where $\hat{\mathcal{T}}$ ranges over triangulations compatible with $S$.

Theorem 3.2. $\operatorname{area}(T) \leq c \cdot M$, for some absolute constant $c$.

Proof. Let $\hat{\mathcal{T}}$ be an arbitrary triangulation compatible with $S$ and let $C$ be a leaf cube of $T$. We can assume $C \cap H$ is not empty. We charge area $(\partial C \cap H)$ to features of $\hat{\mathcal{T}}$, with cases as follows (see Fig. 1):

1. $C / 2 \nsubseteq H$. Necessarily $\partial H$ meets $C / 2$ (and $C$ ). By Lemma 3.3 below, area $(\partial C \cap H)$ is $O(\operatorname{area}(\partial H \cap C))$. We charge $\operatorname{area}(\partial C \cap H)$ to $\partial H \cap C$.

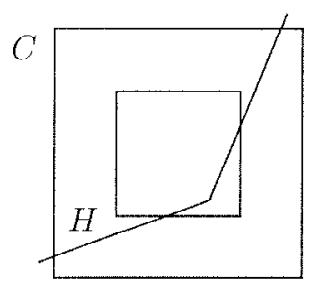

casc 1

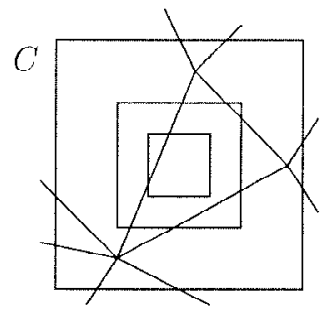

case 2

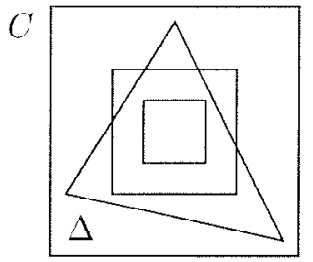

case 3

Fig. 1. Cases of Theorem 3.2. In case 1, $\partial H$ meets $C / 2$; in case 2, the 2-skeleton of $\hat{\mathcal{T}}$ meets $C / 4$; in case 3, the 2-skeleton of $\hat{\mathcal{T}}$ avoids $C / 4$. 
2. $C / 2 \subseteq H$ and $C / 4$ meets the 2 -skeleton of $\hat{\mathcal{T}}$. By Lemma 3.4 below, area $(\partial C \cap H)$ is $O(\operatorname{area}(\hat{\mathcal{T}} \cap C))$. We charge $\operatorname{area}(\partial C \cap H)$ to $\hat{\mathcal{T}} \cap C$.

3. $C / 2 \subseteq H$ and $C / 4$ avoids the 2-skeleton of $\hat{\mathcal{T}}$. This is the most complex case. Let $\alpha, \beta$, and $\gamma$ be constants (chosen in the proof of Lemma 3.6 below, with $0<\alpha, \beta<1$ and $\gamma>1)$. Let the side length of $C$ be $s$. A charging pair $(\Delta, e)$ for $C$ is a triangle $\Delta$ of $\hat{\mathcal{T}}$ and an edge $e$ of $\Delta$ so that area $(\Delta \cap \gamma C) \geq \alpha s^{2}$ and length $(e \cap \gamma C) \geq \beta s$. We charge the area of $\partial C \cap H$ (which is $O\left(s^{2}\right)$ ) to the triangle of a charging pair, whose existence is guaranteed by Lemma 3.6 below. By Lemma 3.8 the total charge to any triangle $\Delta$ of $\hat{\mathcal{T}}$, over all leaf cubes of $\mathcal{T}$, is at most $O(\operatorname{area}(\Delta))$.

In cases 1 and 2 , the charges are to disjoint portions of $\partial H$ and $\hat{\mathcal{T}}$, respectively, so the total charge is $O(\operatorname{area}(\partial H)+\operatorname{area}(\hat{\mathcal{T}}))$ which is $O(\operatorname{area}(\hat{\mathcal{T}}))$. In case 3 , the total charge to any triangle $\Delta$ of $\hat{\mathcal{T}}$ is $O(\operatorname{area}(\Delta))$, so the total charge over all triangles is $O(\operatorname{area}(\hat{\mathcal{T}}))$.

Lemma 3.3. If $C$ is a leaf cube and $C / 2 \nsubseteq H$, then $\operatorname{area}(\partial C \cap H)$ is $O(\operatorname{area}(\partial H \cap C))$.

Proof. Let $s$ be the side length of $C$. Pair each corner of $C / 2$ with the corresponding corner of $C$, and consider the eight subcubes whose opposite corners are formed by the pairs. At least one such subcube $D$ must be entirely outside $H$, else $H$ would contain $C / 2$. Let $d$ be the center of $D$. See Fig. 2 .

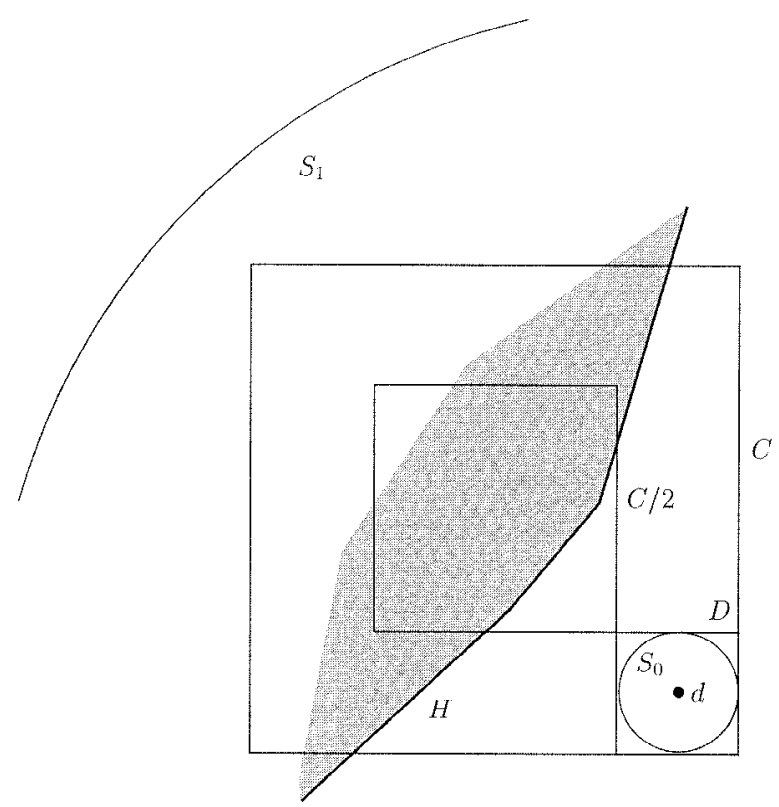

Fig. 2. Proof of Lemma 3.3. 
Let $S_{0}$ and $S_{1}$ be the spheres of radius $s / 8$ and $\sqrt{3} s$, respectively, with center $d . S_{0}$ is contained in $D$ and is outside $H$, and $S_{1}$ contains $C$. Let mappings $\pi_{0}$ and $\pi_{1}$ be central projections from $d$ into $S_{0}$ and $S_{1}$, respectively. Any ray from $d$ to a point of $\partial C \cap H$ must first hit a point of $\partial H \cap C$, since $d$ is outside $H$ and inside $C$. Hence $\pi_{1}(\partial C \cap H) \subseteq \pi_{1}(\partial H \cap C)$. For some constant $c$, we have

$$
c \cdot \operatorname{area}(\partial C \cap H) \leq \operatorname{area}\left(\pi_{1}(\partial C \cap H)\right)
$$

since, for any point $p \in \partial C \cap H$, the angle that a ray $\overrightarrow{d p}$ makes with $\partial C \cap H$ is bounded away from zero, as $d$ is bounded away from $\partial C$. We also have

$$
\begin{aligned}
\operatorname{area}\left(\pi_{1}(\partial C \cap H)\right) & \leq \operatorname{area}\left(\pi_{1}(\partial H \cap C)\right) \\
& =(8 / \sqrt{3})^{2} \cdot \operatorname{area}\left(\pi_{0}(\partial H \cap C)\right) \leq 64 / 3 \cdot \operatorname{area}(\partial H \cap C),
\end{aligned}
$$

proving the lemma.

Lemma 3.4. If $C$ is a leaf cube and $B=C / 2 \subseteq H$ and $B / 2=C / 4$ meets $\hat{\mathcal{T}}^{(2)}$ (the 2-skeleton of $\hat{\mathcal{T}})$, then area $(\partial C \cap H)$ is $O(\operatorname{area}(\hat{\mathcal{T}} \cap C))$.

Proof. Let $s$ be the edge length of $B$. Clearly, area $(\partial C \cap H)$ is $O\left(s^{2}\right)$; we show $\operatorname{area}(\hat{\mathcal{T}} \cap B)$ is $\Omega\left(s^{2}\right)$. Let $D$ be the open cube of side length $s / 2$ centered at a point $x$ of the 2 -skeleton of $\hat{\mathcal{T}}$ in $B / 2$, with the same orientation as $B / 2$; plainly $D \subset B$. Choose three orthogonal edges $e_{1}, e_{2}, e_{3}$ of $\partial D$, and for any $p \in D$ let $l_{i}=l_{i}(p), i=1,2,3$, be the segment through $p$ parallel to $e_{i}$ connecting opposite faces of $D$. We claim at least one of $l_{1}, l_{2}, l_{3}$ must meet $\hat{\mathcal{T}}^{(2)}$. Suppose not, then they must all lie in the same tetrahedron $\Delta$ of $\hat{\mathcal{T}}$, since they share point $p$. Hence the convex hull of $l_{1}, l_{2}, l_{3}$ is contained in $\Delta$, a contradiction since the convex hull contains $x$, which is in $\hat{\mathcal{T}}^{(2)}$.

Let $D_{i}, i=1,2,3$, be the set of points $p \in D$ for which $l_{i}(p)$ meets $\hat{\mathcal{T}}^{(2)}$. Since $D=$ $D_{1} \cup D_{2} \cup D_{3}$, we can assume, say, that the volume of $D_{1}$ is at least $(s / 2)^{3} / 3=s^{3} / 24$. Now $D_{1}$ must be the Cartesian product of a segment of length $s / 2$ parallel to $e_{1}$ with the projection of $\hat{\mathcal{T}}^{(2)} \cap D$ onto a facet of $D$ perpendicular to $e_{1}$. Hence the projection of $\hat{\mathcal{T}}^{(2)}$ has area at least $s^{2} / 12$, and $\hat{\mathcal{T}}^{(2)} \cap D$ itself has at least the same area.

Lemma 3.5. For any leaf cube $C$ of $T, 7 C$ meets the 1-skeleton of $S$.

Proof. Let a refinement step during balancing be the replacement of a leaf node by an interior node with eight children. We show that the lemma holds for $T_{0}$ and is maintained by every refinement step.

By construction, every skewered leaf in $T_{0}$ meets the 1-skeleton of $S$. Every unskewered leaf $C$ in $T_{0}$ has a skewered parent, so certainly $3 C$ meets the 1-skeleton of $S$.

A refinement step maintains the condition of the lemma for every unchanged cube. So suppose $C$ is refined into eight subcubes, with $s$ the original side length of $C$ and $C^{\prime}$ an arbitrary child of $C$. See Fig. 3. $C$ must share a common 2-face with a cube $D$ whose side length is at most $s / 4$. By inductive hypothesis, $7 D$ meets the 1 -skeleton of $S$. Easy calculations show that the $L_{\infty}$ distance between the centers of $C^{\prime}$ and $D$ is at most $7 \mathrm{~s} / 8$ and that $7 D \subset 7 C^{\prime}$. Hence $7 C^{\prime}$ meets the 1-skeleton of $S$. 


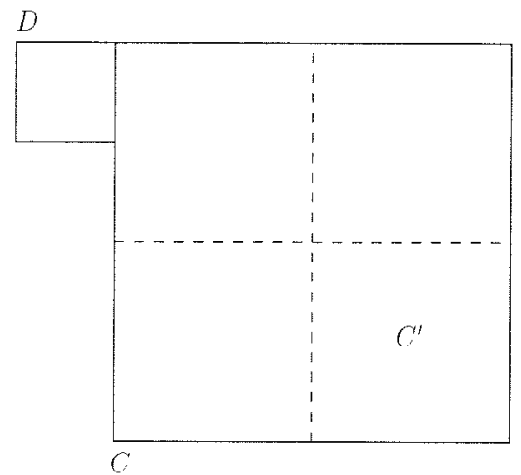

Fig. 3. Proof of Lemma 3.5.

Lemma 3.6. If $C$ is a leaf cube, $B=C / 2 \subseteq H$, and $B / 2$ avoids the 2-skeleton of $\hat{\mathcal{T}}$, then there is a charging pair for $C$.

Proof. Let $s$ be the side length of $B$, so $B / 2$ has side length $s / 2$. By Lemma 3.5 there is a point $q$ on the 1 -skeleton of $\hat{\mathcal{T}}$ within $7 C=14 B$, hence at distance $7 \sqrt{3} s<14 s$ from the center $p$ of $B$. We assume that open segment $p q$ meets the 2 -skeleton of $\hat{\mathcal{T}}$ only at interior points of 2-cells; otherwise a slightly modified argument with a perturbed segment $p^{\prime} q$ is necessary.

Let $\sigma$ be the plane orthogonal to $p q$ through $p$ and let $\pi_{\sigma}$ be the orthogonal projection onto $\sigma$. Let $U$ be the infinite cylinder with axis $p q$ and radius $s / 4$; then the disk $D=U \cap \sigma$ is contained in $B / 2$. Let $p_{1}, \ldots, p_{l-1}$ be the intersections of 2-cells of $\hat{\mathcal{T}}$ with open segment $p q$ in order from $p$ to $q$, and set $p_{0}=p$ and $p_{l}=q$. For $i=1, \ldots, l$, let $\tau_{i}$ be the 3 -simplex of $\hat{\mathcal{T}}$ containing the open segment $p_{i-1} p_{i}$. See Fig. 4 . We have that $B / 2 \subseteq \tau_{1}$, since $p_{0}=p \in B / 2$ and the 2-skeleton of $\hat{\mathcal{T}}$ avoids $B / 2$.

Let $\mu_{i}$ be the connected component of $\partial \tau_{i} \cap U$ containing $p_{i}$. Choose $k$ minimal so that $\mu_{k}$ contains a point of the 1 -skeleton of $\hat{\mathcal{T}}$; such $k$ must exist since $p_{l}=q$ is on the 1-skeleton of $\hat{\mathcal{T}}$.

We claim that $\mu_{i}$ fully cuts $U$, i.e., $\pi_{\sigma}\left(\mu_{i}\right)=D$, for $i=1, \ldots, k$. Now $\mu_{1}$ fully cuts $U$, since $D \subseteq B / 2 \subseteq \tau_{1}$. For $1<i \leq k, \mu_{i-1}$ must be a portion of a single 2-face of $\tau_{i}$, since it contains no point on the 1 -skeleton of $\hat{\mathcal{T}}$. Hence there must be two connected components to $U \cap \partial \tau_{i}$, specifically $\mu_{i-1}$ and $\mu_{i}$, and $\mu_{i}$ must fully cut $U$.

We now show that $\mu_{k}$ lies at distance at most $28 s$ from $\sigma$. Let $f_{k-1}$ and $f_{k}$ be the faces of $\tau_{k}$ containing $p_{k-1}$ and $p_{k}$, and let $P_{k-1}$ and $P_{k}$ be the planes containing $f_{k-1}$ and $f_{k}$, respectively. Now $P_{k-1} \cap D=P_{k-1} \cap U \cap \sigma$ must be empty, since $P_{k-1} \cap U=\mu_{k-1}$ lies entirely in face $f_{k-1}$ but $D=U \cap \sigma$ lies entirely in the interior of simplex $\tau_{1}$. Also $P_{k} \cap D$ must be empty: if not, choose $u \in P_{k} \cap D$; then $p_{k}$ and $u$ lie on opposite sides of $P_{k-1}$ within $U$, so segment $u p_{k}$ meets an interior point of face $f_{k-1}$ of $\tau_{k}$; however, $u p_{k}$ also lies entirely in the plane $P_{k}$ of face $f_{k}$ of $\tau_{k}$, a contradiction. The intersection of $P_{k}$ with $\partial U$ is an ellipse, whose center $p_{k}$ is at distance at most $14 s$ from $\sigma$. The ellipse avoids $D$, since $P_{k}$ avoids $D$. Hence the farthest point of the ellipse is at most $28 s$ from 


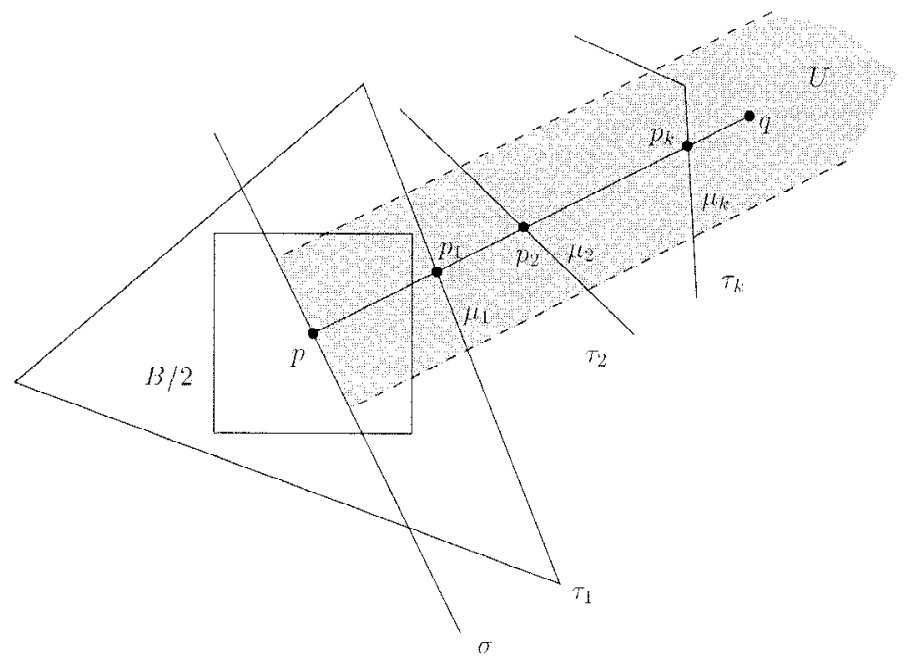

Fig. 4. Proof of Lemma 3.6.

$\sigma$. Since the closed halfspace of $P_{k}$ containing $D$ also contains $\tau_{k}$ and $\mu_{k}, \mu_{k}$ is at most distance $28 s$ from $\sigma$.

We set the charging-pair constants $\alpha=\pi / 48, \beta=\frac{1}{8}$, and $\gamma=43$. To finish the proof, notice that area $\left(\mu_{k}\right) \geq \operatorname{area}\left(\pi_{\sigma}\left(\mu_{k}\right)\right)=\pi(s / 4)^{2}$. Since $\mu_{k}$ consists of portions of at most three triangular faces of $\tau_{k}$, at least one such triangle $\Delta$ satisfies area $(\Delta \cap U) \geq$ $\pi s^{2} / 48=\alpha s^{2} . \Delta$ is the triangle of $\hat{\mathcal{T}}$ promised by the lemma statement. We have $\Delta \cap \bar{U}$ within distance $28 s$ from $\sigma$, in fact within distance $28 s$ from $\sigma \cap U \subset B / 2 \subset B$. Since the side length of $B$ is $s, \Delta \cap U \subset(2 \cdot 28+1) B \subset \gamma C$.

It remains to find a piece of an edge of $\Delta$ of length at least $\beta s$ within $\gamma C$. Let $U^{\prime}$ be the cylinder coaxial with $U$ but with double the radius. By analogous reasoning, we have $\Delta \cap U^{\prime}$ within distance $42 s$ from $\sigma \cap U^{\prime} \subset B$, so $\Delta \cap U^{\prime} \subset(2 \cdot 42+1) B \subset \gamma C$. If $\Delta$ is completely contained within $U^{\prime}$, then by the isoperimetric inequality [16] the perimeter $L$ of $\Delta$ satisfies $L \geq \sqrt{4 \pi \cdot \operatorname{area}(\Delta)} \geq \pi s / 2 \sqrt{3}$, so one edge of $\Delta$ has length at least $\pi s / 6 \sqrt{3} \geq \beta s$. Otherwise $\partial \Delta$ intersects both $\partial U^{\prime}$ and the interior of $U$. Since $\partial U$ and $\partial U^{\prime}$ are separated by $s / 4$, there is a portion of an edge of $\partial \Delta$ lying within $U^{\prime}$ of length at least $s / 8=\beta s$.

The width of a compact planar set $S$, width $(S)$, is the smallest distance $w$ so that $S$ is contained in the closed region between two parallel lines $w$ apart. The diameter of $S$, $\operatorname{diam}(S)$, is the length of the longest segment contained in the convex hull of $S$. It is easy to see that the area of $S$ is at most $\operatorname{width}(S) \cdot \operatorname{diam}(S)$.

Lemma 3.7. Let $\Delta$ be an obstacle triangle and let $r$ be the radius of its inscribed circle. If leaf cube $C$ with side length $s$ has charging pair $(\Delta, e)$, then s is $O(r)$. 
Proof. The width of $\Delta$ is at most $3 r$, by some elementary geometry. Since $(\Delta, e)$ is a charging pair for $C$, we have

$$
\begin{aligned}
\alpha s^{2} & \leq \operatorname{area}(\Delta \cap \gamma C) \\
& \leq \operatorname{width}(\Delta \cap \gamma C) \cdot \operatorname{diam}(\Delta \cap \gamma C) \\
& \leq 3 r \cdot \sqrt{3} \gamma s .
\end{aligned}
$$

Hence $s \leq 3 \sqrt{3} \gamma r / \alpha$, which is $O(r)$.

Lemma 3.8. The total charge to triangle $\Delta$ of $\hat{\mathcal{T}}$ from all leaf cubes in case 3 above is $O(\operatorname{area}(\Delta))$.

Proof. Let $p$ and $r$ be the perimeter and inscribed-circle radius of $\Delta$, respectively. Some elementary geometry gives area $(\Delta)=p r / 2$.

Consider the set $C_{s}$ of all leaf cubes with a fixed side length $s$. Since no two cubes in $C_{s}$ overlap, no point in space is covered by more than $\gamma^{3}$ open supercubes $\gamma C$, for $C \in C_{s}$. Since the intersection of $\gamma C$ with $\partial \Delta$ has length at least $\beta s$, no more than $\gamma^{3} p /(\beta s)=O(p / s)$ cubes in $C_{s}$ can be charged to $\Delta$. The charge for each cube is $O\left(s^{2}\right)$, so the total charge to $\Delta$ for cubes in $C_{s}$ is $O(p s)$.

Let $\hat{s}$ be the largest cube side length for which a cube is charged to $\Delta$; by Lemma 3.7, $\hat{s}$ is $O(r)$. The total charge over all cube sizes is $O\left(p\left(s_{0}+2 s_{0}+\cdots+\hat{s}\right)\right)=O(p \hat{s})=$ $O(p r)$, which is $O(\operatorname{area}(\Delta))$.

\subsection{Triangulating the Tree}

Theorem 3.9. In time $O\left(n^{5}\right)$ it is possible to construct a triangulation $\mathcal{T}$ from the octtree $T$ so that $\operatorname{area}(\mathcal{T}) \leq c^{\prime} \cdot\left(\operatorname{area}(T)+\operatorname{area}(S)+b^{2}\right)$, where $c^{\prime}$ is an absolute constant and $b$ is the side length of the root cube of $T . \mathcal{T}$ has $O\left(n^{5}\right)$ tetrahedra and partitions the convex hull $H$ of $S$.

For $C$ a leaf cube of $T$, the clipped cube $C_{H}$ is $C \cap H$. We choose below a subdivision $\mathcal{P}$ of $H$ whose 3 -cells are clipped-cube interiors and whose 2-skeleton is a triangulation. In Sections 3.5.2 and 3.5.3 we show how to triangulate a clipped cube so that its boundary triangulation matches $\mathcal{P}$. The desired triangulation $\mathcal{T}$ is then obtained simply by pasting the clipped-cube triangulations into $\mathcal{P}$.

In octtree $T$, two adjacent cubes may have overlapping but distinct $k$-faces, $k=1,2$, if the cubes are of different sizes. A clipped-cube $k$-face is minimal if no other clippedcube face of the same dimension is properly contained within it; a minimal $k$-face is a tree-partitioning face if it is properly contained in another clipped-cube face (possibly of higher dimension). It is easy to see that any clipped-cube $k$-face is the union of minimal faces of dimension at most $k$. In fact, since $T$ is balanced, any 1-face is the union of at most four minimal 1-faces and three vertices, and any 2-face is the union of at most four minimal 2-faces, four minimal 1-faces, and a vertex (forming a "+"-shape in the middle of the 2-face). 


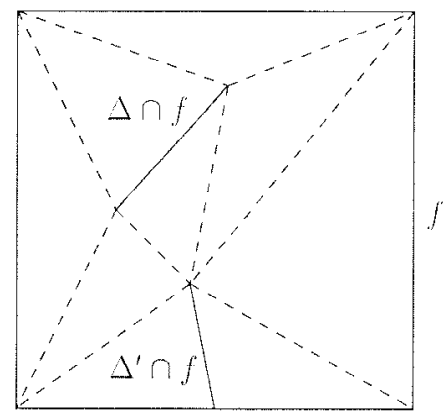

Fig. 5. $\Delta$ and $\Delta^{\prime}$ are obstacle triangles that meet minimal cube 2-face $f$. In $\mathcal{P}, f$ is replaced by a triangulation of $f$ compatible with $\Delta \cap f$ and $\Delta^{\prime} \cap f$.

Let $\mathcal{P}^{\prime}$ consist of clipped-cube interiors plus all minimal 0-, 1-, and 2-faces. Subdivision $\mathcal{P}$ is obtained from $\mathcal{P}^{\prime}$ by replacing 1- and 2-faces. A 1-face $e$ of $\mathcal{P}^{\prime}$ is replaced with the chain of 0 - and 1-cells formed by subdividing $e$ at each point of intersection with an obstacle triangle. Let $f$ be a 2 -face of $\mathcal{P}^{\prime}$. Form the set of line segments obtained by intersecting $f$ with all obstacle triangles, then triangulate this set together with the set of 0 - and 1-cells partitioning $\partial f$. The resulting triangulation replaces $f$ in $\mathcal{P}$. See Fig. 5 . It is easy to check that $\mathcal{P}$ is a subdivision of $H$ whose 2-skeleton has been triangulated.

The combinatorial complexity of leaf cube $C, n_{C}$, is the number of edges of $C$ plus the number of obstacle triangles meeting $C$. It is easy to check that $\operatorname{bdry}\left(C_{H}, \mathcal{P}\right)$ has $O\left(n_{C}\right)$ edges and vertices.

3.5.1. Central Triangulations. Let $Q$ be a 3-cell in a polyhedral subdivision $\mathcal{Q}$ with $\operatorname{bdry}(Q, \mathcal{Q})$ a triangulation and let $q$ be an interior point of $Q$. Recall that by the definition of polyhedral subdivision, $Q$ is convex. The central triangulation of $Q$ from $q$ consists of the tetrahedra formed by $q$ and the triangles in $\operatorname{bdry}(Q, \mathcal{Q})$, and all tetrahedral faces.

Proposition 3.10. If $\operatorname{bdry}(Q, \mathcal{Q})$ has $k$ vertices, then the area of any central triangulation of $Q$ is at most $3 k / 2$ times the area of $\partial Q$.

Proof. Let $q$ be the central triangulation vertex. There are at most $3 k$ edges of $\operatorname{bdry}(Q, \mathcal{Q})$. Each new tetrahedral 2 -face is formed by $q$ and such an edge, and has area at most half the area of $\partial Q$.

3.5.2. Triangulating an Unskewered Cube. For this section, $C$ is an unskewered leaf cube (i.e., $C$ avoids the 1-skeleton of $S$ ). A triangulation $\mathcal{T}_{C}$ of $C_{H}$ is obtained as follows. Start with the subdivision consisting of $C_{H}$ and bdry $\left(C_{H}, \mathcal{P}\right)$. Subdivide $C_{H}$ by all obstacle triangles that meet it. Notice that all such obstacle triangles must cut $C$ fully and cannot meet within $C$; furthermore, $\operatorname{bdry}\left(C_{H}, \mathcal{P}\right)$ already contains all edges of intersection between obstacle triangles and $\partial C_{H}$. Now triangulate any new 2-cells, and centrally triangulate each 3-cell.

Lemma 3.11. $\mathcal{T}_{C}$ has area $O(\operatorname{area}(S \cap C)+\operatorname{area}(\partial C \cap H))$. 


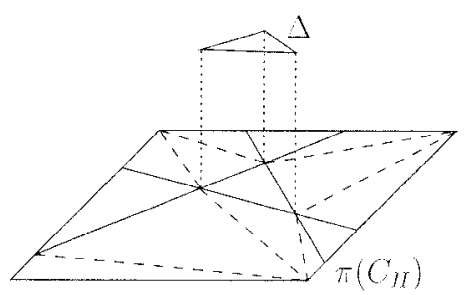

Fig. 6. The edges of obstacle $\Delta$ are projected onto a horizontal plane, extended to lines, and clipped to the projection of $C_{H}$ (labeled $\pi\left(C_{H}\right)$ ). The result is triangulated and each triangle is lifted to an infinite vertical prism.

Proof. Let $\mathcal{T}_{0}$ be the subdivision before central triangulation, and let $C_{0}$ be a 3-cell of $\mathcal{T}_{0}$. We show that the number of vertices in $\operatorname{bdry}\left(C_{0}, \mathcal{T}_{0}\right)$ is constant. The lemma then follows using Proposition 3.10 and the fact that $\operatorname{area}\left(\mathcal{T}_{0}\right)$ is area $(S \cap C)+\operatorname{area}(\partial C \cap H)$.

First notice that $C_{0}$ has at most fourteen polyhedral 2-faces, six faces that are subsets of a 2-face of $C$ and at most eight faces separating a vertex of $C$ from $C_{0}$. Hence $C_{0}$ has a constant number of polyhedral vertices. Any vertex of $\operatorname{bdry}\left(C_{0}, \mathcal{T}_{0}\right)$ that is not a polyhedral vertex must be a cube partitioning vertex, of which there are at most a constant number.

3.5.3. Triangulating a Skewered Cube. For this section, $C$ is a skewered leaf cube (i.e., $C$ meets the 1-skeleton of $S$ ); recall $C$ has side length $s_{0}$. A triangulation $\mathcal{T}_{C}$ of $C_{H}$ is obtained in two steps. We first compute a triangulation $\mathcal{T}_{C}^{\prime}$ so that bdry $\left(\mathcal{T}_{C}^{\prime}\right)=$ $\operatorname{bdry}\left(C_{H}, \mathcal{T}_{C}^{\prime}\right)$ is a refinement of $\operatorname{bdry}\left(C_{H}, \mathcal{P}\right)$. Using Lemma 3.15, we then compute a triangulation $\mathcal{T}_{C}$ so that $\operatorname{bdry}\left(\mathcal{T}_{C}\right)=\operatorname{bdry}\left(C_{H}, \mathcal{P}\right)$.

The first step has four substeps. In the following, the "vertical" direction can be chosen to be any direction not parallel to a face of $C_{H}$ or a face of $S$ and so that no vertical line meets three obstacle edges that do not already meet at a common vertex. See Fig. 6.

1. Orthogonally project each edge of $\operatorname{bdry}\left(C_{H}, \mathcal{P}\right)$ onto a horizontal plane (i.e., a plane orthogonal to the vertical direction), and extend the projection to a line. Similarly project and extend each obstacle edge meeting $C$.

2. Let $\mathcal{T}_{2}$ be a (two-dimensional) triangulation of the resulting arrangement, truncated to the projection of $C_{H}$. Lift $\mathcal{T}_{2}$ to a set of infinite vertical triangular prisms.

3. Subdivide $C_{H}$ using both the vertical prisms and any obstacle triangles meeting $C$, forming a subdivision $\mathcal{T}_{3}$.

4. Extend the triangulation of $\operatorname{bdry}\left(C_{H}, \mathcal{P}\right)$ to form a triangulation of the 2-skeleton of $\mathcal{T}_{3}$. Then centrally triangulate each 3 -cell, forming the triangulation $\mathcal{T}_{C}^{\prime}$.

Lemma 3.12. The total length of $\mathcal{T}_{2}$ is $O\left(n_{C} s_{0}\right)$.

Proof. Let $\ell$ be a line in the plane and let $t(\ell)$ be the number of edges of $\mathcal{T}_{2}$ met by $\ell$. Consider the arrangement (in substep 2 above) before triangulation and truncation. By the zone theorem for lines [8], the total combinatorial complexity of the cells of the arrangement intersected by a line $\ell$ is $O\left(n_{C}\right)$, so $t(\ell)$ is $O\left(n_{C}\right)$. 
Let $\mu$ be the rigid-motion invariant measure on sets of lines in the plane [16]; up to a constant multiple, the length of $\mathcal{T}_{2}$ is $\int t(\ell) d \mu$. The measure of the set of lines intersecting $\mathcal{T}_{2}$ is $O\left(s_{0}\right)$, since the perimeter of $\mathcal{T}_{2}$ is $O\left(s_{0}\right)$ [16]. Hence $\int t(\ell) d \mu$ is bounded by $O\left(s_{0} n_{C}\right)$.

Lemma 3.13. Triangulation $\mathcal{T}_{C}^{\prime}$ has area $O\left(\operatorname{area}(S \cap C)+\operatorname{area}(\partial C \cap H)+n_{C} s_{0}^{2}\right)$.

Proof. The total area of the vertical 2-cells in the subdivision $\mathcal{T}_{3}$ is $O\left(n_{C} s_{0}^{2}\right)$, since the total length of $\mathcal{T}_{2}$ is $O\left(n_{C} s_{0}\right)$ and the height of $C_{H}$ is at most $O\left(s_{0}\right)$. Any nonvertical 2-cell is a portion of either $S \cap C$ or $\partial C \cap H$, hence the total area of all nonvertical 2-cells is area $(S \cap C)+\operatorname{area}(\partial C \cap H)$. Let $P$ be a 3-cell in $\mathcal{T}_{3}$; we show that the number of vertices in $\operatorname{bdry}\left(P, \mathcal{T}_{3}\right)$ is constant. The lemma then follows using Proposition 3.10 and the bound on the sum of the areas of all 2-cells.

Cell $P$ is a section of a triangular prism and has at most five polyhedral facets, hence a constant number of polyhedral vertices. We claim bdry $\left(P, \mathcal{T}_{3}\right)$ has at most two additional vertices per vertical polyhedral edge of $P$. To see this, note that, by construction of $\mathcal{T}_{3}$, any vertex of $\operatorname{bdry}\left(P, \mathcal{T}_{3}\right)$ must either be a vertex of $\operatorname{bdry}\left(C_{H}, \mathcal{T}_{3}\right)$, and hence a polyhedral vertex of $P$, or must lie in the interior of a collinear vertical chain of edges of $\mathcal{T}_{3}$. In the latter case, the vertex must either lie in the interior of an obstacle triangle, and hence must be a polyhedral vertex of $P$, or on the closure of an obstacle edge. However, there can be at most two such vertices of this last type per vertical chain, since the vertical direction was chosen so that no three obstacle edges lie on a common vertical line.

It is easy to see that $\mathcal{T}_{C}^{\prime}$ has $O\left(n_{C}^{3}\right)$ tetrahedra, has $O\left(n_{C}^{2}\right)$ vertices on $\operatorname{bdry}\left(\mathcal{T}_{C}^{\prime}\right)$, and can be computed in time $O\left(n_{C}^{3}\right)$.

The second step is to transform $\mathcal{T}_{C}^{\prime}$ into $\mathcal{T}_{C}$ by removing the vertices of $\operatorname{bdry}\left(C_{H}, \mathcal{T}_{C}^{\prime}\right)$ not in bdry $\left(C_{H}, \mathcal{P}\right)$. As will be seen in the proof of Lemma 3.15, removing a vertex that lies in the interior of a 2-face of $\operatorname{bdry}\left(C_{H}, \mathcal{P}\right)$ is slightly different from removing a vertex that lies in the interior of an edge of $\operatorname{bdry}\left(C_{H}, \mathcal{P}\right)$.

Let $G=(V, E)$ be the 1-skeleton of $\operatorname{bdry}\left(C_{H}, \mathcal{T}_{C}^{\prime}\right)$ as a graph, and similarly let $G_{P}=\left(V_{P}, E_{P}\right)$ be the 1-skeleton of $\operatorname{bdry}\left(C_{H}, \mathcal{P}\right)$. A subset of $V$ is independent if no two vertices are connected by an edge of $E$.

Lemma 3.14. There is an independent subset of $V \backslash V_{P}$ whose size is a constant fraction of $V \backslash V_{P}$ so that each vertex has constant degree in $G$.

Proof. The average degree of vertices in the subgraph of $G$ induced by $V \backslash V_{P}$ is at most six, by planarity. Any vertex $v$ in $V \backslash V_{P}$ is incident to at most four vertices of $V_{P}$ : either $v$ lies in the interior of a triangle of $\operatorname{bdry}\left(C_{H}, \mathcal{P}\right)$, in which case it can be incident to at most three vertices of $V_{P}$, or $v$ lies on an edge of bdry $\left(C_{H}, \mathcal{P}\right)$, in which case it can be incident to at most four vertices of $V_{P}$. Hence the average degree of vertices $V \backslash V_{P}$ in $G$ is at most ten. By standard techniques [14], it follows that there is a bounded-degree independent set whose size is a constant fraction of $V \backslash V_{P}$.

Lemma 3.15. There is a triangulation $\mathcal{T}_{C}$ of $C$ with $\operatorname{bdry}\left(\mathcal{T}_{C}\right)=\operatorname{bdry}\left(C_{H}, \mathcal{P}\right) . \mathcal{T}_{C}$ can 
be computed from $\mathcal{T}_{C}^{\prime}$ in time $O\left(n_{C}^{3}\right)$, adding $O\left(n_{C}^{3}\right)$ tetrahedra and area $O\left(s_{0}^{2} \log n_{C}+\right.$ $\operatorname{area}(S \cap C))$.

Proof. $\mathcal{T}_{C}^{\prime}$ is updated in stages, with the final stage yielding $\mathcal{T}_{C}$. Each stage removes the independent set of vertices guaranteed by Lemma 3.14. Clearly, the number of stages is $\log$ arithmic in the number of vertices of $\mathcal{T}_{C}^{\prime}$, i.e., $O\left(\log n_{C}\right)$.

For simplicity, we first assume that every vertex to be removed lies in the interior of some 2-face of bdry $\left(C_{H}, \mathcal{P}\right)$ and not on an edge of $\operatorname{bdry}\left(C_{H}, \mathcal{P}\right)$. Consider the triangles incident to $v$; all such triangles are coplanar. Let $S_{v}$ be the polygon formed by the triangle edges opposite $v$. It is possible to push $v$ slightly inside $C_{H}$ maintaining the combinatorial structure of $\mathcal{T}_{C}^{\prime}$ (in particular, $v$ must not be pushed through the plane of the face opposite $v$ in any tetrahedron incident to $v$ ). Pushing $v$ leaves a dimple in the triangulation of $C_{H}$. The dimple can be filled by triangulating $S_{v}$ and then adding to $\mathcal{T}_{C}^{\prime}$ the tetrahedra formed by $v$ and the new triangles.

The area added to $\mathcal{T}_{C}^{\prime}$ at each stage is at most $O\left(s_{C}^{2}\right)$, where $s_{C}$ is the side length of cube $C$. To see this, first note that when a vertex $v$ is pushed, the change in area of the tetrahedra incident to $v$ is negligible, since the perturbation of $v$ can be made arbitrarily small. The area of the new tetrahedral faces is at most a constant times the area of $S_{v}$, since $S_{v}$ has at most a constant number of edges. Since the vertices at each stage form an independent set, the polygons $\left\{S_{v}\right\}$ have disjoint interiors and their total area is at most the surface area of $C$, i.e., $O\left(s_{C}^{2}\right)$. Hence the total area added at all stages is $O\left(s_{C}^{2} \log n_{C}\right)$.

Now suppose some vertex $v$ lies on an edge $e$ of $\operatorname{bdry}\left(C_{H}, \mathcal{P}\right)$. Edge $e$ may be a portion of a polyhedral edge of $\partial C_{H}$ or may be interior to a polyhedral face of $\partial C_{H}$. If $v$ does not lie on an obstacle triangle, then the approach is similar, except that the triangulation of $S_{v}$ must use a piece of edge $e$.

Now suppose $v$ lies on an edge $e$ of $\operatorname{bdry}\left(C_{H}, \mathcal{P}\right)$ and also on an obstacle triangle. It does not suffice simply to push $v$ into the interior of $C_{H}$ as above, since the resulting triangulation would not be compatible with the obstacles. Instead, vertex $v$ is removed as follows. Necessarily $v$ lies in the interior of a segment $s$ of the intersection of the obstacle triangle with $\partial C_{H}$. Let $p$ and $q$ be the vertices on either side of $v$ along $s$. Let $\Delta_{1}, \ldots, \Delta_{k}$ be the obstacle triangles incident to $s$ that enter the interior of $C_{H}$, in cyclic order around $s$. We can assume $k>0$, i.e., not all triangles incident to $s$ lie on $\partial C_{H}$, otherwise $v$ can be perturbed as before. Let $P_{i}$ be the plane through $\Delta_{i}$, and choose the positive and negative open halfspaces of $P_{i}$ so that the positive halfspace contains $\Delta_{1}, \ldots, \Delta_{i-1}$ and the negative halfspace contains $\Delta_{i+1}, \ldots, \Delta_{k}$. Let $S_{v}$ be defined as above, and let $S_{v}^{+}$be the polygon bounded by $p q$ and the portion of $S_{v}$ in the positive halfspace of $P_{1}$; similarly, let $S_{v}^{-}$be the polygon bounded by $p q$ and the portion of $S_{v}$ in the negative halfspace of $P_{k}$. For each triangle $\Delta_{i}, i=1, \ldots, k-1$, in turn, split $v$ into two vertices $v$ and $v_{i}$ connected by an edge, and perturb $v_{i}$ slightly into the interior of $\partial C_{H}$ while staying on $\Delta_{i}$. See Fig. 7. Incidences to $v$ are adjusted as follows. Within the positive halfspace of $P_{i+1}$, any edge, triangle, or tetrahedron previously incident to $v$ should be made incident to $v_{i}$; in the negative halfspace of $P_{i+1}$, incidences remain with $v$. Incidences to $v$ on $P_{i+1}$ expand by a dimension: an edge $u v$ on $P_{i+1}$ becomes a triangle $u v v_{i}$; a triangle $t u v$ on $P_{i+1}$ becomes a tetrahedron $t u v v_{i}$. Finally, for the last triangle $\Delta_{k}$, simply perturb $v_{k}=v$ to the interior of $\partial C_{H}$ while staying on $\Delta_{k}$. After all splitting and perturbation there is again a dimple on $\partial C_{H}$. Specifically, the perturbation of $v_{1}$ 


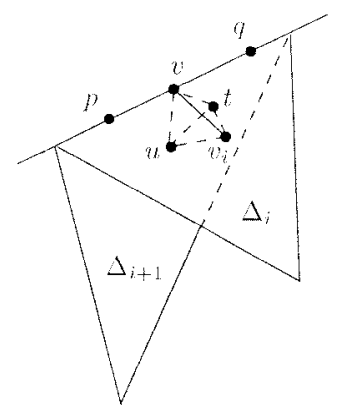

Fig. 7. Vertex $v$ lies on $\partial C_{H}$ (not shown); both $\Delta_{i}$ and $\Delta_{i+1}$ are interior to $C_{H} ; v_{i}$ lies on $\Delta_{i} ; u$ and $t$ lie on $\Delta_{i+1}$ though they need not be close to $v$.

formed a dimple bounded by $S_{v}^{+}$; each subsequent perturbation of $v_{i}, i>1$, increased the dimple by the tetrahedron $p q v_{i} v_{i-1}$; in addition, the final perturbation of $v=v_{k}$ increased the dimple by $S_{v}^{-}$. To fill the dimple, choose triangulations of $S_{v}^{-}$and $S_{v}^{+}$; then add the tetrahedra formed by $v_{1}$ and the triangles of $S_{v}^{+}$, the tetrahedra $p q v_{i} v_{i-1}$, $i=2, \ldots, k$, and the tetrahedra formed by $v_{k}$ and the triangles of $S_{v}^{-}$.

To check that the resulting triangulation is compatible with all obstacles, observe that the perturbation of $v_{i}$ maintains a two-dimensional triangulation of obstacle $\Delta_{i}$, with the exception of the triangle $p q v_{i}$. However, triangle $p q v_{i}$ is a face of tetrahedron $p q v_{i} v_{i-1}$ (and tetrahedron $p q v_{i+1} v_{i}$ ) and hence is added when the dimple is filled.

The perturbation increases area by adding tetrahedra $p q v_{i} v_{i+1}$, by replacing an edge $u v$ with a triangle $u v v_{i}$, and by replacing a triangle $t u v$ with a tetrahedron $t u v v_{i}$. In the first two cases, the additional area is negligible since the distances $v v_{i}$ can be made arbitrarily small. In the last case, the triangle $t u v$ is essentially duplicated, adding the triangle $t u v_{i}$. However, notice that after the entire perturbation, neither $v$ in its new position at $v_{k}$ nor any vertex split from $v$ lies on $\partial C_{H}$. Hence over all stages each triangle $t u v$ can be duplicated at most three times, once per vertex. Since $t u v$ is part of an obstacle lying in $C_{H}$, the total additional area is $O\left(\operatorname{area}\left(S \cap C_{H}\right)\right)$.

The number of tetrahedra required to fill the dimple resulting from perturbing $v$ is constant if $v$ is not on an obstacle and $O\left(n_{C}\right)$ if $v$ is on an obstacle. Since $O\left(n_{C}^{2}\right)$ vertices have to be removed, the total number of tetrahedra over all stages is $O\left(n_{C}^{3}\right)$. New tetrahedra are also created by expanding a triangle to a tetrahedron. However, this happens at most once per vertex of each triangle, hence at most $O\left(n_{C}^{3}\right)$ times altogether.

The running time of the algorithm is $\mathrm{O}\left(n_{C}^{3}\right)$, since it takes time $O\left(n_{C}^{2}\right)$ to find independent sets and $O\left(n_{C}^{3}\right)$ to add tetrahedra.

\subsubsection{Accounting}

Lemma 3.16. $\sum_{C} n_{C} s_{0}^{2}=O\left(\operatorname{area}(S)+b^{2}\right)$, where the sum runs over all skewered leaf cubes $C$ and $b$ is the side length of the root bounding cube.

Proof. Let $m_{C}$ be the total number of obstacle triangles that intersect cube $C$. Since every skewered cube meets some obstacle triangle, $n_{C}$ is $O\left(m_{C}\right)$. We can express $\sum_{C} m_{C} s_{0}^{2}$ 
as

$$
\sum_{\Delta \in S}\left|S_{\Delta}\right| s_{0}^{2}
$$

where $S_{\Delta}$ is the set of skewered leaf cubes intersected by obstacle triangle $\Delta$. We can write

$$
\left|S_{\Delta}\right|=a_{\Delta}+m_{\Delta},
$$

where $a_{\Delta}$ and $m_{\Delta}$ are respectively the number of cubes $C$ in $S_{\Delta}$ so that the doubled cube $2 C$ avoids or meets the boundary of $\Delta$.

Consider first cubes $C$ in $S_{\Delta}$ so that $2 C$ avoids the boundary of $\Delta$. Plainly the area of $2 C \cap \Delta$ is $\Theta\left(s_{0}^{2}\right)$. Since no point in space is covered by more than eight open doubled skewered cubes, $a_{\Delta} \leq 8 \cdot \operatorname{area}(\Delta) / \Omega\left(s_{0}^{2}\right)=O\left(\operatorname{area}(\Delta) / s_{0}^{2}\right)$. Hence $a_{\Delta} s_{0}^{2}$ is $O(\operatorname{area}(\Delta))$, and $\sum_{\Delta \in S} a_{\Delta} s_{0}^{2}$ is $O(\operatorname{area}(S))$.

Now consider cubes $C$ in $S_{\Delta}$ so that $2 C$ meets the boundary of $\Delta$. The boundaries of the doubled cubes lie on the planes of a cubic grid of step size $s_{0}$. An obstacle triangle edge $e$ meets at most $3\left\lceil\right.$ length $\left.(e) / s_{0}\right\rceil$ planes, which is $O\left(b / s_{0}\right)$ (recall that $b$ is the side length of the box bounding the obstacle set $S$ ). Hence each edge meets $O\left(b / s_{0}\right)$ doubled cubes, so $m_{\Delta}$ is $O\left(b / s_{0}\right)$. We have

$$
\sum_{\Delta \in S} m_{\Delta} s_{0}^{2}=O\left(n \cdot b / s_{0} \cdot s_{0}^{2}\right)=O\left(n b s_{0}\right)=O\left(b^{2}\right)
$$

using $b /(2 n)<s_{0} \leq b / n$.

Proof of Theorem 3.9. By Lemmas 3.11, 3.13, and 3.15, the total area of $\mathcal{T}$ is

$$
\sum_{C} \operatorname{area}(\partial C \cap H)+\sum_{C} \operatorname{area}(S \cap C)+\sum_{C} n_{C} s_{0}^{2},
$$

where the first two summations run over all leaf cubes and the last over skewered leaf cubes. The first two summations add to $O(\operatorname{area}(T)+\operatorname{area}(S))$. Using Lemma 3.16, the last summation is $O\left(\operatorname{area}(S)+b^{2}\right)$.

Since the minimum cube size is about $b / n$, the total number of leaf cubes is $O\left(n^{3}\right)$. Both the running time to compute $\mathcal{T}$ and the number of tetrahedra are bounded by $O\left(\sum_{C} n_{C}^{3}\right)=O\left(\sum_{C}\left(m_{C}+1\right)^{3}\right)=O\left(\sum_{C} m_{C}^{3}\right)+O\left(n^{3}\right)$, where $m_{C}$ is the number of triangles meeting cube $C$. All leaf cubes $C$ have faces lying on a cubic grid of planes with step size $s_{0}$; a triangle can hit at most $O\left(n^{2}\right)$ such grid cubes and thus at most $O\left(n^{2}\right)$ leaf cubes of $T$. Hence the number of incidences between leaf cubes and triangles, $\sum_{C} m_{C}$ is $O\left(n^{3}\right)$. The sum $\sum_{C} m_{C}^{3}$ is maximized if $m_{C}$ is as large as possible, i.e., $n$, for as many cubes $C$ as possible, i.e., $O\left(n^{2}\right)$, yielding an upper bound of $O\left(n^{3} \cdot n^{2}\right)=O\left(n^{5}\right)$.

\subsection{General Obstacle Sets}

Theorem 3.17. Let $S$ be an arbitrary obstacle set with a total of $n$ simplices. In time $O\left(n^{6}\right)$ it is possible to compute a triangulation compatible with $S$ whose area is within a constant factor of the smallest possible. The triangulation has $O\left(n^{6}\right)$ tetrahedra and partitions the convex hull of $S$. 
We begin the proof by finding a bounding box of $S$. Choose a diametrical segment of the convex hull $H$ of $S$. Project $H$ onto a plane orthogonal to the segment, and choose a smallest square containing the projection. $S$ fits into a box $B_{S}$ which is a translation of the Cartesian product of the diametrical segment with the square. As before, perturb $B_{S}$ slightly to guarantee that no obstacle face is parallel to a face of $B_{S}$. Let $B_{S}$ have size $h \times h \times \ell h$. We can assume that $\ell$ is an integer. It is easy to see that the area of $\partial H$ is $\Omega\left(\ell h^{2}\right)$.

We choose a family $\mathcal{B}$ of boxes that partition $B_{S}$. Conceptually split $B_{S}$ into $\ell$ consecutive $h \times h \times h$ cubes. Any cube that contains a vertex of $S$ is a box in $\mathcal{B}$. Any maximal union of consecutive cubes not containing a vertex is also a box in $\mathcal{B}$. Clearly, there are at most $2 v-1$ boxes in $\mathcal{B}$, where $v$ is the number of vertices in $S$.

Refine the obstacle set $S$ using the planes separating adjacent boxes in $\mathcal{B}$, as follows. For each plane $P$, if $P$ hits an edge $e$ of $S$, split the edge into two subedges and the vertex $e \cap P$; if $P$ hits a 2-simplex $\Delta$ of $S$, split the simplex into two 2-faces and the edge $e \cap \Delta$; finally, triangulate the region $P \cap H$ compatibly with all intersection vertices $e \cap P$ and intersection edges $\Delta \cap P$, and add all resulting 2-faces to $S$. After examining all planes, triangulate any remaining 2-face that is not a triangle. Let $S^{\prime}$ be the refined obstacle set and let $S_{B}$ be the subset of $S^{\prime}$ lying inside box $B$. Recall $|\mathcal{T}|$ is the area of triangulation $\mathcal{T}$. Let $M=\inf _{\mathcal{T}}|\mathcal{T}|$, where $\mathcal{T}$ varies over all triangulations compatible with $S$; for $B \in \mathcal{B}$, let $M_{B}=\inf _{\mathcal{T}}|\mathcal{T}|$, where $\mathcal{T}$ varies over all triangulations compatible with $S_{B}$.

Lemma 3.18. $\sum_{B \in \mathcal{B}} M_{B}=O(M)$.

Proof. Let $\hat{\mathcal{T}}$ be an arbitrary triangulation compatible with $S$. Use each plane $P$ that separates adjacent boxes in $\mathcal{B}$ to refine $\hat{\mathcal{T}}$, as follows: if $P$ hits $0-, 1-$, or 2 -cells, then they are refined as $S$ was refined to $S^{\prime}$; if $P$ hits a 3-cell $\Delta$, then $\Delta$ is split into two 3-cells and the 2-cell $P \cap \Delta$. The area of $P \cap \Delta$ is $O\left(h^{2}\right)$, for a total over all planes of $O\left(|\mathcal{B}| \cdot h^{2}\right)=O(\operatorname{area}(\partial H))=O(\operatorname{area}(\hat{\mathcal{T}}))$. Each 3 -cell in the resulting subdivision is either a tetrahedron or has a constant number of polyhedral faces; in the latter case the cell can be centrally triangulated (after triangulating any nontriangular faces), increasing area by at most a constant factor. The resulting triangulation can be split into a triangulation of each box $B \in \mathcal{B}$, with triangulation areas summing to $O(\operatorname{area}(\hat{\mathcal{T}}))$.

The obstacle set $S^{\prime}$ is triangulated by triangulating each set $S_{B}$ in turn, and then pasting the resulting triangulations together. A detail is that adjacent triangulations must be compatible along their common boundary; however, this is easily guaranteed using the technique of Section 3.5. Choose a box $B \in \mathcal{B}$ of size $h \times h \times \ell_{B} \cdot h, \ell_{B} \geq 1$ an integer. Let $L_{B}$ be the affine transformation that fixes one of the square sides of $B$ and shrinks the orthogonal direction by a factor of $\ell_{B}$; then $L_{B}(B)$ is a cube. Triangulate $L_{B}(B)$ using $L_{B}\left(S_{B}\right)$ as before: build an octtree with root cube $L_{B}(B)$ and subdivide it using the obstacles $L_{B}\left(S_{B}\right)$, as described in Section 3.3, and then triangulate each octtree leaf cube as in Section 3.5, again using $L_{B}\left(S_{B}\right)$. Apply the inverse transformation $L_{B}^{-1}$ to obtain a triangulation $\mathcal{T}_{B}$ compatible with $S_{B}$.

Let $T_{B}$ be the image under $L_{B}^{-1}$ of the octtree, then $L_{B}\left(T_{B}\right)$ is the octtree with root cube $L(B)$ and $T_{B}$ is an octtree-like structure formed from blocks with side-length ratio 
$1 \times 1 \times \ell_{B}$. The area of $T_{B}$, area $\left(T_{B}\right)$, is $\sum_{C}$ area $(C \cap H)$, where the sum runs over all leaf boxes $C$ in $T_{B}$.

Lemma 3.19. $\operatorname{area}\left(T_{B}\right)=O\left(M_{B}\right)$.

Proof. We can assume that $\ell_{B}>1$, otherwise the lemma follows from Theorem 3.2. Choose a leaf box $C$ in $T_{B}$ and conceptually partition it into $\ell_{B}$ consecutive cubes. We show below that, for each such cube $D, 21 D$ meets the 1 -skeleton of $S_{B}$. Let $\hat{\mathcal{T}}$ be an arbitrary triangulation of $S_{B}$. Using the same argument as the proof of Theorem 3.2, we charge $\operatorname{area}(D \cap H)$ to features of $\hat{\mathcal{T}}$ (with an appropriate modification to the chargingpair constants, since $21 D$ rather than $7 D$ meets the 1 -skeleton). Since area $(C \cap H)$ is bounded by $\sum_{D}$ area $(D \cap H), D$ in the partition of $C$, the lemma follows.

By Lemma 3.5, $7 L_{B}(C)$ meets the 1-skeleton of $L_{B}\left(S_{B}\right)$, so $7 C$ meets the 1-skeleton of $S_{B}$. Since $S$ has no vertices within $B, 7 C$ must meet an edge $e$ of $S_{B}$ with endpoints on opposite square faces of $B$. Consider the subsegment $e^{\prime}$ of $e$ lying between the planes through the square faces of $C$. Clearly, there is a translate of $7 C$ that contains $e^{\prime}$ and overlaps $7 C$. Hence for any of the cubes $D$ partitioning $C, 21 D$ meets $e^{\prime}$.

Lemma 3.20. $\ell_{B} \cdot \operatorname{area}\left(L_{B}\left(T_{B}\right)\right)=O\left(\operatorname{area}\left(T_{B}\right)\right)$ and $\ell_{B} \cdot \operatorname{area}\left(L_{B}\left(S_{B}\right)\right)=O\left(\operatorname{area}\left(S_{B}\right)\right)$.

Proof. We show the second statement; the first is easier. Assume $\ell_{B}>1$, otherwise the lemma is trivial. Choose triangle $\Delta \in S_{B}$. Since $B$ contains no vertices of $S, \Delta$ must result from refining a triangle of $S$ by the planes through the two square sides of $B$. Hence $\Delta$ is a triangle, with an edge $e$ on one square side of $B$ and a vertex on the opposite square side of $B$. Similarly, $L_{B}(\Delta)$ has an edge $e^{\prime}$ of the same length as $e$ on one side of $L_{B}(B)$, and a vertex on the opposite side. The height of $\Delta$ opposite $e$ is at least $h \ell_{B}$, and the height of $L_{B}(\Delta)$ opposite $e^{\prime}$ is at most $h \sqrt{3}$. Hence $\ell_{B} \cdot \operatorname{area}\left(L_{B}(\Delta)\right)=$ $O(\operatorname{area}(\Delta))$.

Lemma 3.21. $\operatorname{area}\left(\mathcal{T}_{B}\right)=O\left(\operatorname{area}\left(T_{B}\right)+\operatorname{area}\left(S_{B}\right)+\ell_{B} \cdot h^{2}\right)$.

Proof. By Theorem 3.9, we have $\operatorname{area}\left(L_{B}\left(\mathcal{T}_{B}\right)\right)=O\left(\operatorname{area}\left(L_{B}\left(T_{B}\right)\right)+\operatorname{area}\left(L_{B}\left(S_{B}\right)\right)+\right.$ $\left.h^{2}\right)$. Hence

$$
\begin{aligned}
\operatorname{area}\left(\mathcal{T}_{B}\right) & =O\left(\ell_{B} \cdot \operatorname{area}\left(L_{B}\left(\mathcal{T}_{B}\right)\right)\right) \\
& =O\left(\ell_{B} \cdot \operatorname{area}\left(L_{B}\left(T_{B}\right)\right)+\ell_{B} \cdot \operatorname{area}\left(L_{B}\left(S_{B}\right)\right)+\ell_{B} h^{2}\right) \\
& =O\left(\operatorname{area}\left(T_{B}\right)+\operatorname{area}\left(S_{B}\right)+\ell_{B} h^{2}\right),
\end{aligned}
$$

using Lemma 3.20.

Proof of Theorem 3.17. By Lemmas 3.19 and 3.21, the triangulation $\mathcal{T}_{B}$ of each box $B \in \mathcal{B}$ has area $O\left(M_{B}+\ell_{B} \cdot h^{2}\right)$. Hence the whole triangulation has area

$$
\sum_{B \in \mathcal{B}} O\left(M_{B}\right)+\sum_{B \in \mathcal{B}} O\left(\ell_{B} \cdot h^{2}\right)=O(M)+O\left(\ell h^{2}\right)=O(M),
$$


using Lemma 3.18 and $\ell h^{2}=O(\operatorname{area}(\partial H))=O(M)$. The bounds on running time and tetrahedra follow from Theorem 3.9 since there are $O(n)$ boxes in $\mathcal{B}$.

\subsection{Worst-Case Bounds}

For obstacle set $S$ in $\mathbb{R}^{3}$, recall that $c(S)=\inf _{\mathcal{T}} \operatorname{area}(\mathcal{T}) /$ area $(S)$, where $\mathcal{T}$ varies over all triangulations compatible with $S$. The following lemma gives worst-case bounds on $c(S)$. As before, $S$ must contain faces partitioning the boundary of its convex hull.

Lemma 3.22. For any wide obstacle set $S$ in $\mathbb{R}^{3}, c(S)=O(\sqrt{n})$, where $n$ is the number of simplices in $S$. There is a wide set $\hat{S}$ of $O(n)$ obstacles with $c(\hat{S})=\Omega(\sqrt{n})$.

Proof. Let $B$ be the minimum-size bounding cube of $S$, perturbed slightly so that no obstacle face is parallel to a face of $B$. Let $B$ have side length $b$. Split $B$ into a grid of identical cubes, where each cube has side length $s \approx b / \sqrt{n}$, so there are about $n^{3 / 2}$ cubes altogether. Using the algorithms of Section 3.5, triangulate each clipped cube and paste the triangulations together, yielding a triangulation $\mathcal{T}$ compatible with $S . \mathcal{T}$ has area $O\left(n^{3 / 2} \cdot s^{2}\right)=O\left(b^{2} \sqrt{n}\right)$ (the surface area of the cubes) plus $O(\operatorname{area}(S))$ plus $O\left(\sum_{C} n_{C} s^{2}\right)$, where $n_{C}$ is the combinatorial complexity of cube $C$. An analysis similar to Lemma 3.16 shows that $\sum_{C} n_{C} s^{2}$ is $O\left(\operatorname{area}(S)+n \cdot(b / s) \cdot s^{2}\right)=O\left(\operatorname{area}(S)+b^{2} \sqrt{n}\right)$. Hence the ratio area $(\mathcal{T}) / \operatorname{area}(S)$ is $O(\sqrt{n})$, as $\operatorname{area}(S)$ is $\Omega\left(b^{2}\right)$.

For the second statement, choose an axis-aligned unit cube $B$. Subdivide $B$ into a cubical grid of about $\sqrt{n} \times \sqrt{n} \times \sqrt{n}$ identical subcubes. For each one-dimensional row of subcubes parallel to the $x$-, $y$-, or $z$-axis, choose a very thin obstacle triangle that covers all the subcube centers in the row. Slightly perturb the resulting set of $\Theta(n)$ triangles so that no two intersect. Add to the obstacle triangles a triangulation of their convex hull, forming the obstacle set $\hat{S}$. Since the 1-skeleton of $\hat{S}$ passes very near the center of each subcube, Lemma 3.4 implies that any triangulation $\mathcal{T}$ compatible with $\hat{S}$ must have area $\Omega\left((1 / \sqrt{n})^{2}\right)=\Omega(1 / n)$ within each subcube. Since there are $n^{3 / 2}$ subcubes, the total area of $\mathcal{T}$ is $\Omega(\sqrt{n})$. Since the area of the convex hull is $O(1)$ and the area of the remaining obstacles can be made arbitrarily small, $c(\hat{S})=\Omega(\sqrt{n})$.

An argument similar to this proof shows that if $S$ contains $n$ points (and the faces of the convex hull), then $c(S)=O\left(n^{1 / 3}\right)$. Furthermore, there is a set $\hat{S}$ of $n$ points with $c(\hat{S})=\Omega\left(n^{1 / 3}\right)$. These results contrast with the results of Agarwal et al. [2]. They show there is a set $S$ of $n$ points in $\mathbb{R}^{3}$ so that in any triangulation of $S$, some line meets $\sqrt{n}$ triangulation faces. Similarly there is a set $S$ of $n$ obstacle triangles in $\mathbb{R}^{3}$ so that in any triangulation compatible with $S^{\prime}$, some line meets $\Omega(n)$ triangulation triangles, even though it misses all obstacles.

\section{Discussion}

We have not tried to estimate the approximation ratio for the construction in Section 3. Our algorithm is based on Eppstein's algorithm, which approximates the minimum-length 
Steiner triangulation of a set of points in two dimensions. Eppstein is able to prove an approximation ratio of 316 (though he suspects the true ratio is much smaller, perhaps around 20); our proof is much less careful about constants than his. A challenging open problem is to construct triangulations of approximately minimum weight in two or three dimensions with reasonable constants and with a reasonable number of vertices.

In two dimensions, the minimum spanning tree provides an intrinsic measure of the minimum weight triangulation, in the sense that their weights differ by at most a logarithmic factor (Lemma 2.2). In three dimensions, the surface area of the octtree constructed in Section 3 is an intrinsic measure of the minimum weight triangulation (Theorem 3.2). It would be of interest to obtain a more natural intrinsic measure.

The analysis of ray-shooting-by-walking can be extended to other subdivisions besides triangulations. For example, consider the leaf cubes of the octtree constructed in Section 3, with the modification that each unskewered cube is partitioned by all obstacles that cut it fully. Label each skewered leaf cube with the number of obstacle triangles that meet it, and label all the other 3-cells 1 . Then it is possible to walk through the partitioned octtree along a line $\ell$ with total cost proportional to $w(\ell)$, where $w(\ell)$ is the sum of the labels of the 3-cells intersected by $\ell$. The analysis in Section 3 shows that $\int w(\ell) d \mu$ is the area of the minimum weight triangulation, to within a constant factor. Two problems arise naturally when considering alternative subdivisions for ray-shooting queries: first, to determine if the area on the minimum weight triangulation is always a relevant bound; second, to provide an analytic comparison of the constants that arise from different subdivisions.

\section{References}

1. P. Agarwal, Range searching, in CRC Handbook of Discrete and Computational Geometry, J. E. Goodman and J. O'Rourke, eds., CRC Press, Boca Raton, FL, 1997, pp. 575-598.

2. P. Agarwal, B. Aronov, and S. Suri, Stabbing triangulations by lines in 3D, Proc. Eleventh Ann. Symp. on Computational Geometry, 1995, pp. 267-276.

3. P. Agarwal and J. Matoušek, On range searching with semialgebraic sets, Discrete Comput. Geom. 11:393418, 1994.

4. P. Agarwal and M. Sharir, Applications of a new partition scheme, Discrete Comput. Geom. 9:11-38, 1993.

5. R. Beirouti and J. Snoeyink, Implementations of the LMT heuristic for minimum weight triangulation, Proc. Fourteenth Ann. Symp. on Compuational Geometry, 1998, pp. 96-105.

6. M. Bern and D. Eppstein, Approximation algorithms for geometric problems, in Approximation Algorithms for NP-Hard Problems, D. Hochbaum, ed., PWS, Boston, MA, 1997, pp. 296-345.

7. K. Clarkson, Approximation algorithms for planar traveling salesman tours and minimum-length triangulations, Proc. 2nd ACM-SIAM Symp. on Discrete Algorithms, 1991, pp. 17-23.

8. H. Edelsbrunner, Algorithms in Combinatorial Geometry, Springer-Verlag, Berlin, 1987.

9. D. Eppstein, Approximating the minimum weight Steiner triangulation, Discrete Comput. Geom., 11:163$191,1994$.

10. S. Fortune, A beam-tracing algorithm for prediction of indoor radio propagation, in Applied Computational Geometry: Towards Geoemtric Engineering, M. Lin and D. Manocha, eds., LNCS 1148, Springer-Verlag, Berlin, 1996, pp. 157-166.

11. J. Hershberger and S. Suri, A pedestrian approach to ray shooting: shoot a ray, take a walk, J. Algorithms 18:403-431, 1995.

12. J. S. B. Mitchell, D. M. Mount, and S. Suri, Query-sensitive ray shooting, Internat. J. Comput. Geom. Appl. 7(4):317-347, 1997. 
13. D. W. Moore, Simplicial Mesh Generation with Applications, Ph.D. Thesis, Report 92-1322, Dept. of Computer Science, Cornell University, 1992.

14. J. O'Rourke, Computational Geometry in C, Cambridge University Press, Cambridge, 1994.

15. M. Pellegrini, Ray-shooting and lines in space, in CRC Handbook of Discrete and Computational Geometry, J. E. Goodman and J. O’Rourke, eds., CRC Press, Boca Raton, FL, 1997, pp. 599-614.

16. L. Santaló, Integral Probability and Geometric Probability, Encyclopedia of Mathematics and its Applications, Volume 1, Addison-Wesley, Reading, MA, 1979.

Received February 3, 1997, and in revised form August 21, 1998. 